\section{L'abolition du}

registre des armes

d'épaule : le rôle

potentiel des

principes non écrits

avoir lieu dans les provinces. Laisser trop de latitude au Parlement en laisse moins à Vancouver, Regina, Toronto et Québec d'agir dans l'intérêt de leurs populations respectives, écartant ainsi les principes fondamentaux de fédéralisme et de démocratie. Les Canadiens ont le droit de débattre dans leurs capitales, dans leurs lieux de travail, dans leurs communautés et dans leurs maisons de l'usage approprié des fruits d'une collaboration intergouvernementale. Il en va de même pour des groupes d'intérêt public, qui ne devraient pas être limités à un seul forum fédéral pour exprimer leurs opinions.

\title{
Introduction
}

L'appel devant la Cour suprême dans l'affaire Canada (Procureur général) $c$ Québec (Procureur général) ${ }^{1}$, soulève une question d'interprétation constitutionnelle: est-ce que sa compétence en matière de droit criminel permet au Parlement d'abroger une loi et d'un seul coup détruire unilatéralement toutes les données recueillies sous l'égide de cette même $1 \mathrm{i}^{2}$ ? Notamment, cette question se pose dans le contexte d'une collaboration entre le fédéral et les provinces dans un domaine, la réglementation des armes à feu, ayant un double aspect.

En répondant à cette question interprétative, il est nécessaire d'appliquer les principes non écrits identifiés par la Cour suprême - surtout le fédéralisme et la démocratie - dans un contexte constitutionnel dans lequel le fédéralisme coopératif est privilégié, voire encouragé. L'auteur a argumenté ailleurs que la destruction des données est ultra vires du Parlement parce que le caractère véritable de la disposition législative en question est « la propriété et les droits civils dans la province », une compétence provinciale ${ }^{3}$. Le but du présent texte est d'analyser le rôle potentiel des principes de fédéralisme et démocratie.

Donner une étendue large au pouvoir fédéral en l'espèce coupe court au débat qui devrait

Étant donné que la coopération entre les deux paliers de gouvernement est de mise, il incombe aux tribunaux de sanctionner les gestes unilatéraux nocifs à la collaboration intergouvernementale. On ne peut pas d'une part encourager de l'action collaborative, mais d'autre part laisser à un seul acteur le pouvoir unilatéral de détruire les fruits de cette coopération.

Décider autrement mènerait à une situation absurde qui irait à l'encontre du principe de fédéralisme coopératif. Au lieu de collaborer sur une base de respect mutuel, les provinces et le fédéral devraient présumer que l'autre n'agirait pas de bonne foi. Pour ce qui est du registre des armes à feu, par exemple, les provinces auraient dû maintenir des registres parallèles contenant exactement les mêmes données. Présumer de la mauvaise foi est la seule façon de se protéger de la destruction unilatérale d'un registre bâti conjointement, mais une telle présomption mine le fédéralisme coopératif.

Les données du registre des armes à feu n'appartiennent pas à un seul titulaire, mais à tous les Canadiens. Compte tenu du travail acharné au fil des ans des Canadiens et de leurs agents, le Parlement et l'exécutif fédéral ne peuvent pas agir tout seuls. Il est plutôt nécessaire soit de renvoyer les données aux provinces pour qu'elles prennent elles-mêmes une décision finale après un débat public, soit de coopérer avec 
elles afin de décider d'une façon collaborative du sort du registre des armes à feu. Il ne serait permissible de détruire les données qu'après un dialogue collaboratif avec ceux et celles qui ont travaillé au fil des ans à bâtir le registre.

Notre analyse nous mène à deux conclusions importantes. D'une part, la Cour suprême doit interpréter d'une façon étroite le pouvoir fédéral en matière de droit criminel en l'espèce. D'autre part, la Cour suprême doit reconnaître une obligation d'agir de bonne foi lorsque les deux paliers de gouvernement ont collaboré afin de bâtir un régime réglementaire.

\section{Interprétation constitutionnelle}

Aujourd'hui, la doctrine constitutionelle canadienne se trouve loin des compartiments étanches de compétence établis par le Conseil privé. La Cour suprême a retracé les lignes de la séparation des pouvoirs entre le fédéral et le provincial en mettant l'accent sur la coopération. La réponse à la question d'interprétation posée en l'espèce en dira long sur l'avenir du droit canadien et surtout sur la collaboration intergouvernementale par rapport aux enjeux ayant, comme la réglementation des armes à feu, un double aspect.

Les principes non écrits sont incontournables dans ce contexte ${ }^{4}$. Ils «guident l'interprétation du texte et la définition des sphères de compétence, la portée des droits et obligations ainsi que le rôle de nos institutions politiques $»^{5}$. En l'espèce, les principes aident à « combler les vides des dispositions expresses du texte constitutionnel » ${ }^{6}$ parce qu'il est question de la portée du pouvoir fédéral relatif au droit criminel: «Les tribunaux définissent ainsi graduellement la portée des domaines de compétence pertinents $\gg$.

Le contexte constitutionnel est aussi d'une importance capitale :
[L]'interprétation de ces compétences et de leur articulation doit être évolutive et adaptée aux réalités politiques et culturelles changeantes de la société canadienne [...] Le fonctionnement même du régime fédéral canadien doit donc continuellement faire l'objet de nouvelles analyses au regard des valeurs fondamentales qu'il était destiné à favoriser ${ }^{8}$.

En l'espèce, les principes fondamentaux de démocratie et fédéralisme mènent à une interprétation étroite du pouvoir réclamé par le Parlement, un pouvoir qui n'est pas compatible avec un contexte constitutionnel dominé par le fédéralisme coopératif.

\section{Principes fondamentaux: démocratie et fédéralisme}

Reconnaître comme la Cour d'appel que le gouvernement fédéral n'ait ni à coopérer ni à consulter avec les provinces avant de détruire des données quant aux biens et droits civils dans les provinces donne un sens trop large à la compétence fédérale en matière de droit criminel et nuit à l'opportunité laissée aux individus et à des groupes d'intérêt public d'être écoutés et entendus dans les forums établis par la Constitution.

La destruction des données mandatée par le Parlement a privé divers individus et groupes de leurs moyens de s'exprimer dans un débat public au Québec et ailleurs, bien que ces forums publics soient garantis par la Constitution, dont la démocratie est une considération interprétative essentielle. En agissant ainsi d'une façon unilatérale, le Parlement a outrepassé ses limites constitutionnelles. Il incombe à la Cour suprême d'interpréter l'étendue du pouvoir réclamé par le fédéral à la lumière des principes fondamentaux de démocratie et fédéralisme.

Dans le Renvoi relatif à la sécession $d u$ Québec, la Cour suprême s'est exprimée ainsi : 
Le principe du fédéralisme est une reconnaissance de la diversité des composantes de la Confédération et de l'autonomie dont les gouvernements provinciaux disposent pour assurer le développement de leur société dans leurs propres sphères de compétence. La structure fédérale de notre pays facilite aussi la participation à la démocratie en conférant des pouvoirs au gouvernement que l'on croit le mieux placé pour atteindre un objectif sociétal donné dans le contexte de cette diversité ${ }^{9}$.

Préserver une zone de discussion publique s'avère depuis longtemps une préoccupation des tribunaux canadiens. Le système de gouvernement canadien est un système de "government resting ultimately on public opinion reached by discussion and the interplay of ideas ${ }^{10}$. La libre discussion des affaires d'importance publique «is the breath of life for parliamentary institutions ${ }^{11}$ parce qu' « un peuple souverain exerce son droit à l'autonomie gouvernementale à travers le processus démocratique $»^{12}$.

Comme l'a constaté le juge Cannon dans l'affaire Re Alberta Statutes :

\begin{abstract}
Democracy cannot be maintained without its foundation: free public opinion and free discussion throughout the nation of all matters affecting the State within the limits set by the criminal code and the common $l a w^{13}$.
\end{abstract}

Une large place pour la libre discussion est intimement liée au bon fonctionnement de la démocratie canadienne et joue ainsi un rôle important dans l'interprétation constitutionnelle:

$[P]$ ublic opinion, in order to meet such a responsibility, demands the condition of a virtually unobstructed access to and diffusion of ideas. Parliamentary government postulates a capacity in men, acting freely and under self-restraints, to govern themselves; and that advance is best served in the degree achieved of individual liberation from subjective as well as objective shackles [...] This constitutional fact is the political expression of the primary condition of social life, thought and its communication by language. Liberty in this is little less vital to man's mind and spirit than breathing is to his physical existence ${ }^{14}$.

Cette préoccupation qui a marqué l'interprétation des limites des compétences législatives demeure fondamentale dans l'ère de la Charte canadienne des droits et libertés ${ }^{15}$. Selon le juge en chef Dickson, «[1]es tribunaux doivent être guidés par des valeurs et des principes essentiels à une société libre et démocratique, lesquels comprennent [...] la foi dans les institutions sociales et politiques qui favorisent la participation des particuliers et des groupes dans la société $»{ }^{16}$.

Le fait que l'abolition du registre ait été promise dans une plateforme électorale ne change rien à cette analyse : une promesse politique ne peut conférer une compétence constitutionnelle. Qui plus est, le principe de démocratie tel qu'élaboré par la Cour suprême reconnait qu'au Canada, les débats politiques ne sont pas limités à ceux tenus à la Chambre des communes et au Sénat. Pour que la vie démocratique canadienne soit saine, l'implication des individus, des groupes d'intérêt public et des politiciens dans les forums établis par la Constitution, y compris les assemblées provinciales, est nécessaire. La démocratie est plus large dans la fédération canadienne qu'ailleurs, ce qui convient aux individus et groupes d'intérêt public qui auront toujours plusieurs autres forums où ils peuvent se faire entendre si le Parlement n'est pas à l'écoute.

Les Canadiens ont le droit de s'exprimer lors d' « un processus permanent de discussion $\gg$ partout au pays, non seulement à la Chambre des communes :

Les rapports entre démocratie et fédéralisme signifient par exemple que peuvent coexister des majorités différentes et également 
légitimes dans divers provinces et territoires ainsi qu'au niveau fédéral. Aucune majorité n'est plus, ou moins, «légitime» que les autres en tant qu'expression de l'opinion démocratique, quoique, bien sûr, ses conséquences varieront selon la question en jeu. Un système fédéral de gouvernement permet à différentes provinces de mettre en \{oe\}uvre des politiques adaptées aux préoccupations et aux intérêts particuliers de leur population. En même temps, le Canada dans son ensemble est aussi une collectivité démocratique au sein de laquelle les citoyens poursuivent et réalisent des objectifs à l'échelle nationale, par l'intermédiaire d'un gouvernement fédéral agissant dans les limites de sa compétence. La fonction du fédéralisme est de permettre aux citoyens de faire partie simultanément de collectivités différentes et de poursuivre des objectifs aussi bien au niveau provincial qu'au niveau fédéral ${ }^{18}$.

Ce processus de discussion à plusieurs niveaux "entraîne des compromis, des négociations et des délibérations » menant aux «meilleures solutions aux problèmes publics $\gg$.

\section{Contexte constitutionnel: fédéralisme coopératif ${ }^{20}$}

Le fédéralisme coopératif encadre l'analyse constitutionnelle dans le présent appel. Comme l'a remarqué le juge Iacobucci, «le Canada (plus particulièrement celui d'aprèsguerre) a été érigé » sur «la théorie du fédéralisme coopératif ${ }^{21}$. La doctrine canadienne «a rejeté l'approche formaliste rigide pour favoriser les efforts d'accommodements et de coopération entre les gouvernements ${ }^{22}$ tant que l'interprétation constitutionnelle canadienne «tend vers les notions plus souples du double aspect et du fédéralisme coopératif $»^{23}$.

Rappelons que le registre des armes à feu doit son existence à la doctrine de double aspect $^{24}$. La loi sur les armes à feu ${ }^{25}$ a instauré un système réglementaire des biens tenus dans les provinces afin d'obtenir l'objectif de sécurité publique :

Les dispositions relatives aux permis obligent quiconque possède une arme à feu à obtenir un permis. Les dispositions relatives à l'enregistrement exigent l'enregistrement de toutes les armes à feu. La combinaison des deux parties du régime vise à assurer que, lorsqu'une arme à feu change de propriétaire, le nouveau propriétaire ait un permis. Sans système d'enregistrement, cela serait impossible à vérifier. Si une arme à feu est trouvée en la possession d'une personne sans permis, le système d'enregistrement permet au gouvernement d'en déterminer la provenance. Avec un régime d'enregistrement en place, les propriétaires détenant un permis peuvent être tenus responsables de la cession de leurs armes. Le système d'enregistrement vise aussi, comme l'ensemble de la loi, à réduire l'usage abusif. Lorsqu'une personne est déclarée coupable d'un crime de violence ou qu'il lui est interdit de posséder une arme, le régime d'enregistrement est censé aider la police à déterminer si le contrevenant possède en fait une arme à feu et à la confisquer. Le régime $\mathrm{d}$ 'enregistrement vise également à réduire la contrebande et le commerce illégal des armes à feu. Ces liens multiples démontrent que les dispositions de la Loi sur les armes à feu relatives à l'enregistrement et aux permis sont tous deux étroitement liées au but visé par le Parlement, soit la promotion de la sécurité par la réduction de l'usage abusif de toutes les armes à feu ${ }^{26}$.

En l'absence des trois critères d'une loi criminelle valide, ce système réglementaire serait probablement tombé plutôt sous la compétence provinciale en matière de propriété et droits civils.

Les deux paliers de gouvernement ainsi que les citoyens canadiens ont donc collaboré afin de développer un registre qui répondait au besoin d'améliorer la sécurité publique. Des organismes fédéraux et provinciaux ont même signé des ententes de 
«partenariat ${ }^{27}$. Les conclusions de fait du juge de première instance sont claires :

Le Tribunal doit reconnaître un effet réel et concret à cette volonté, clairement exprimé par le Canada et le Québec, de soumettre l'ensemble des renseignements recueillis à deux juridictions, et ce de façon concurrente, ce qui doit signifier, en pratique, quelque chose. [O]n constate, à la lecture du préambule de l'Accord qu'il existe un partenariat entre la GRC et le Québec pour assurer l'administration efficace de la $\mathrm{LAF}^{28}$.

Maintenant le Parlement fédéral prétend qu'il détient le pouvoir de détruire unilatéralement ces données. Il faut analyser cette prétention à la lumière de l'émergence du principe de fédéralisme coopératif.

Par exemple, sans avoir donné son imprimatur aux fruits d'une telle initiative, cette Cour a suggéré dans le Renvoi relatif à la Loi sur les valeurs mobilières qu' « une recherche coopérative de solutions qui satisfont les besoins tant de l'ensemble du pays que de ses composantes ${ }^{29}$ serait préférable à une action fédérale unilatérale pour ce qui était « une matière qui comporte à la fois des aspects nationaux et des aspects locaux ${ }^{30}$ en laissant entendre que « chacun peut collaborer avec l'autre pour s'acquitter de ses responsabilités ${ }^{31}$ :

\footnotetext{
Une telle approche s'inscrit dans le droit fil des principes constitutionnels canadiens et des pratiques adoptées par le fédéral et les provinces dans d'autres sphères d'activité. Ces régimes ont pour pivot le respect par chacun des champs de compétence de l'autre et la collaboration pour principe directeur. Le fédéralisme qui sous-tend le cadre constitutionnel canadien n'exige pas moins ${ }^{32}$.
}

L'exécutif fédéral et les provinces travaillent actuellement afin d'établir une telle commission sur une base collaborative. À quoi sert le principe de fédéralisme coopératif si, par exemple, une commission pan-canadienne des valeurs mobilières mise sur pied après de longues négociations de l'Atlantique au Pacifique opère sous l'épée de Damoclès, toujours menacée d'abolition suite à une décision prise unilatéralement à Ottawa? Donner une portée large au pouvoir fédéral d'agir unilatéralement en l'espèce entraînerait des conséquences néfastes, en réduisant grandement la possibilité des actions coopératives futures.

On est loin de la décision de la Cour suprême dans le Renvoi relatif au Régime d'assistance publique du Canada (C.-B. $)^{33}$, où dans un obiter le juge Sopinka a accordé une marge d'action unilatérale considérable au Parlement. Les contextes factuels et juridiques sont toutefois forts différents. Le régime en question n'impliquait que la provision des fonds par le fédéral : la mise en œuvre des programmes en question était une responsabilité carrément provinciale. Le registre des armes d'épaule, par contre, implique un travail collaboratif entre le fédéral et les provinces. Et juridiquement, la donne a grandement changé. Les mots du juge Sopinka sont tombés avant que la Cour mette l'accent sur l'importance du fédéralisme coopératif. La Cour nous a récemment rappelé par exemple que «[n]otre société devient plus complexe, et pour être efficace, la réglementation exige de plus en plus la coopération des régimes fédéral et provincial interreliés $»^{34}$.

Toutefois, sans une manifestation concrète dans la doctrine constitutionnelle, il sera difficile d'inciter la collaboration qui est devenue de plus en plus nécessaire. Ainsi, il incombe dans un premier temps à la Cour suprême de ne pas accorder une telle latitude au Parlement. Il faut interpréter la compétence fédérale en matière de droit criminel d'une façon qui respecte les principes de démocratie et fédéralisme; la Cour suprême ne devrait pas reconnaître un vaste pouvoir de détruire des données recueillies lors d'un partenariat intergouvernemental. 
Deuxièmement, il est temps de considérer une approche constitutionnelle souple qui imposerait certaines conditions lorsque le fédéral et les provinces travaillent en partenariat, surtout dans un domaine ayant un double aspect.

\section{Conclusion: vers une obligation de bonne foi}

Une façon de garantir aux acteurs qui bâtissent un organisme réglementaire coopératif que leurs efforts dureront serait de reconnaître formellement en droit canadien une obligation de bonne foi. Un semblable devoir de loyauté est déjà reconnu dans d'autres fédérations comme un principe fondamental sans lequel les efforts collaboratifs seraient toujours menacés d'abolition par un geste unilatéral ${ }^{35}$. Au Canada, une telle obligation s'appliquerait aux organismes réglementaires créés coopérativement dans un domaine de double aspect.

Cette obligation aurait deux aspects, un aspect négatif et un aspect positif. L'aspect négatif imposerait une contrainte sur l'action gouvernementale. Un gouvernement ne devrait pas invoquer une compétence constitutionnelle afin de nuire à un autre palier gouvernemental. En l'espèce, malheureusement, on constate que le gouvernement fédéral n'a pas agi de façon conforme à ce principe. Non seulement les ministres fédéraux ont annoncé qu'ils voulaient en partie empêcher les provinces d'établir leurs propres registres ${ }^{36}$, mais l'effet concret de la destruction des données est de rendre extrêmement difficile la construction d'un nouveau registre par un acteur provincial $^{37}$.

L'aspect positif imposerait une obligation de consultation préalable à une action qui minerait l'efficacité d'un régime coopératif. Il y a une analogie appropriée à faire avec la doctrine développée par la Cour suprême dans le contexte des relations avec la Couronne et les Premières nations. Lorsqu'il est question d'une atteinte aux droits autochtones, un devoir de consultation s'impose $^{38}$. Cette obligation est variable : «le niveau de consultation et d'accommodement nécessaire est propor- tionnel à la solidité de la revendi- cation et à la gravité de l'incidence négative que la mesure gouvernementale proposée aurait sur le droit revendiqué $\gg 39$. Une chose est toutefois claire: «le principe de l'honneur de la Couronne commande que celle-ci agisse de bonne foi et tienne une véritable consultation, qui soit appropriée eu égard aux circonstances $» 40$. L'absence de consultation en l'espèce justifierait un recours approprié, soit une consultation, soit une compensation financière ${ }^{41}$, soit tout simplement le transfert des données.

Je ne suggère pas que la violation du principe de fédéralisme rend ultra vires l'action législative en l'espèce ${ }^{42}$. Les principes non écrits servent donc en l'espèce un objectif interprétatif menant dans un premier temps vers une interprétation étroite de la compétence fédérale en matière de droit criminel, ayant pour résultat que la destruction des données est ultra vires du Parlement et dans un deuxième temps, vers l'imposition d'un devoir de consultation $-\mathrm{y}$ compris une obligation de bonne foi préalable à une action gouvernementale qui nuirait au fonctionnement d'un régime collaboratif.

\section{Notes}

* Paul Daly est professeur adjoint, Faculté de droit, Université de Montréal.

1 Canada (Procureur général) c Québec (Procureur général), 2013 QCCA 1138.

2 Loi modifiant le Code criminel et la Loi sur les armes à feu, LC 2012, c 6, art 29 :

(1) Le commissaire aux armes à feu veille à ce que, dès que possible, tous les registres et fichiers relatifs à l'enregistrement des armes à feu autres que les armes à feu prohibées ou les armes à feu à autorisation restreinte qui se trouvent dans le Registre canadien des armes à feu, ainsi que toute copie de ceux-ci qui relève de lui soient détruits.

(2) Chaque contrôleur des armes à feu veille à ce que, dès que possible, tous les registres et 
fichiers relatifs à l'enregistrement des armes à feu autres que les armes à feu prohibées ou les armes à feu à autorisation restreinte qui relèvent de lui, ainsi que toute copie de ceux-ci qui relève de lui soient détruits.

$3 \quad$ Loi constitutionnelle de 1867 (R-U), 30 \& 31 Vict, c 3, reproduite dans LRC 1985, ann II, $\mathrm{n}^{\circ}$ 5, art 92(13). Voir Paul Daly, « Dismantling Regulatory Structures: Canada's Long-Gun Registry as Case Study », 32:2 NJCL [à paraître en 2014].

4 Eugénie Brouillet, «La dilution du principe fédératif et la jurisprudence de la Cour suprême du Canada » (2004), 45:1 C de D 7; JeanFrançois Gaudreault-DesBiens, «The "Principle of Federalism" and the Legacy of the Patriation and Quebec Veto References » (2011), 54 Sup Ct L Rev 78; Jean Leclair, « The Supreme Court of Canada's Understanding of Federalism: Efficiency at the Expense of Diversity » (2003), 28:2 Queen's LJ 411.

5 Renvoi relatif à la sécession du Québec, [1998] 2 RCS 217 au para 52.

6 Renvoi relatif à la rémunération des juges de la Cour provinciale de I.P.E.; Renvoi relatif à l'indépendance et à l'impartialité des juges de la Cour provinciale de I.P.E., [1997] 3 RCS 3 au para 104.

$7 \quad$ Banque canadienne de l'Ouest c Alberta, [2007] 2 RCS 3, 2007 CSC 22 au para 31.

$8 \quad$ Ibid au para 23.

9 Renvoi relatif à la sécession du Québec, supra note 5 au para 58.

10 Saumur v City of Quebec, [1953] 2 SCR 299 à la p 330 (le juge Rand).

11 Reference Re Alberta Statutes - The Bank Taxation Act; The Credit of Alberta Regulation Act; and the Accurate News and Information Act, [1938] SCR 100 à la p 133 [Re Alberta Statutes].

12 Renvoi relatif à la sécession du Québec, supra note 5 au para 64 .

13 Re Alberta Statutes, supra note 11 à la p 146.

14 Switzman v Elbing and A.G. of Quebec, [1957] SCR 285à la p 306 (le juge Rand).

15 Charte canadienne des droits et libertés, partie I de la Loi constitutionnelle de 1982, constituant l'annexe B de la Loi de 1982 sur le Canada (R-U), 1982, c 11.

16 R c Oakes, [1986] 1 RCS 103 au para 64.

17 Renvoi relatif à la sécession du Québec, supra note 5 au para 68.

$18 \quad$ Ibid au para 66.
19 Ibid au para 68.

20 Voir généralement Johanne Poirier, « Fédéralisme coopératif, souveraineté parlementaire et armes à feux : les juges changeront-ils leur fusil d'épaule ? », RDUS [à paraître en 2014].

21 Husky Oil Operations Ltd. c Ministre du Revenu national, [1995] 3 RCS 453 au para 162.

22 Renvoi relatif à la Loi sur les valeurs mobilières, [2011] 3 RCS 837, 2011 CSC 66 au para 58.

23 Canada (Procureur général) c PHS Community Services Society, [2011] 3 RCS 134, 2011 CSC 44 au para 70.

24 Renvoi relatif à la Loi sur les armes à feu (Can.), [2000] 1 RCS 783, 2000 CSC 31 au para 52.

25 Loi sur les armes à feu, LC 1995, c 39.

26 Renvoi relatif à la Loi sur les armes à feu (Can.), supra note 24 au para 47.

27 Québec (Procureur général) c Canada (procureur général), 2012 QCCS 4202 aux paras 9,73 et 76.

$28 \quad$ Ibid au para 83.

29 Renvoi relatif à la Loi sur les valeurs mobilières, supra note 22 au para 132.

$30 \quad$ Ibid au para 131.

31 Ibid.

32 Ibid au para 133.

33 Renvoi relatif au Régime d'assistance publique du Canada (C.-B.), [1991] 2 RCS 525.

34 Nation Tsilhqot'in c Colombie-Britannique, 2014 CSC 44 au para 148.

35 Voir Marcus Klamert, The Principle of Loyalty in EU Law, Oxford, Oxford University Press, 2014.

36 Citons par exemple les propos de la secrétaire parlementaire du ministre de la Justice, Kerry Lynne D. Findlay : "Nous tiendrons notre promesse, et cela suppose que nous agissons de la bonne façon en veillant à ce qu'aucun autre gouvernement ne puisse utiliser l'information pour établir l'inefficace registre des armes d'épaule ». Québec (Procureur général) c Canada (Procureur général), supra note 27 au para 139.

37 Ibid au para 142 : «Les effets juridiques et pratiques de l'article 29 de C-19 se révélant identiques : la destruction des données empêche leur transfert et leur utilisation pour toute province. Cela heurte de plein fouet le désir du 
Québec de maintenir un registre à jour pour les armes d'épaules ».

38 Nation haïda c Colombie-Britannique (Ministre des Forêts), [2004] 3 RCS 511, 2004 CSC 73.

39 Nation Tsilhqot'in c Colombie-Britannique, supra note 34 au para 79.

40 Nation haïda c Colombie-Britannique (Ministre des Forêts), supra note 38 au para 41.

41 Québec (Procureur général) c Canada (Procureur général), 2013 QCCA 1263 au para 40.

42 Colombie-Britannique c Imperial Tobacco Canada Ltée, [2005] 2 RCS 473, 2005 CSC 49. 